\title{
Statistical analysis of stereopsis in ophthalmology research
}

\author{
Teresa Ferrer-Blasco $\cdot$ David Madrid-Costa • \\ Santiago García-Lázaro • Alejandro Cerviño • \\ Robert Montés-Micó
}

Received: 2 May 2011 / Accepted: 4 May 2011 / Published online: 24 May 2011

(C) Springer-Verlag 2011

\section{Dear Editor,}

We appreciate the interest shown by Peyman et al. in our recent paper entitled "Stereopsis in bilaterally multifocal pseudophakic patients"[1]. Peyman et al. suggest that stereoacuity outcomes should be not analyzed as mean values, considering the values given by some stereotests. As discussed in our paper, Titmus and random dot stereotests provide fixed disparity levels, hence accuracy of the outcome measures depends on the number of steps and their disparity level. In contrast, the Howard-Dolman method gives a continuous measure of stereoacuity with high accuracy. Continuous measures using the HowardDolman method provide appropriate stereoacuity values that can be analyzed as means and/or standard deviations for statistical purposes. In addition to this, some values obtained by different methods used in ophthalmology research differ considerably in step size, and their corresponding graphical representation may prove difficult for an appropriate comparison analysis. In these cases, the use of a log scale may be of use to aid in that comparison. From a statistical point of view, if two groups of values differ statistically ( $p$-values, power, confidence intervals, etc.) that would equally occur regardless of the linear or logarithmic character of the scale.

\section{Reference}

1. Ferrer-Blasco T, Madrid-Costa D, García-Lázaro S, Cerviño A, Montés-Micó R (2011) Stereopsis in bilaterally multifocal pseudophakic patients. Graefes Arch Clin Exp Ophthalmol 249:245-251

T. Ferrer-Blasco $\cdot$ D. Madrid-Costa $\cdot$ S. García-Lázaro •

A. Cerviño $\cdot$ R. Montés-Micó $(\bowtie)$

University of Valencia,

Valencia, Spain

e-mail: robert.montes@uv.es 